\title{
Growth of Intermediate-Mass Black Holes in Globular Clusters
}

\author{
Kayhan Gültekin \\ M. Coleman Miller \\ Douglas P. Hamilton \\ University of Maryland, College Park, Dept. of Astronomy
}

\begin{abstract}
We present results of numerical simulations of sequences of binary-single scattering events of black holes in dense stellar environments. The simulations cover a wide range of mass ratios from equal mass objects to 1000:10:10 $M_{\odot}$ and compare purely Newtonian simulations to simulations in which Newtonian encounters are interspersed with gravitational wave emission from the binary. In both cases, the sequence is terminated when the binary's merger time due to gravitational radiation is less than the arrival time of the next interloper. We find that black hole binaries typically merge with a very high eccentricity $(0.93 \leq e \leq 0.95$ pure Newtonian; $0.85 \leq e \leq 0.90$ with gravitational wave emission) and that adding gravitational wave emission decreases the time to harden a binary until merger by $\sim 30$ to $40 \%$. We discuss the implications of this work for the formation of intermediate-mass black holes and gravitational wave detection.
\end{abstract}

Subject headings: black hole physics — galaxies: star clusters — globular clusters: general — stellar dynamics

\section{Introduction}

Recent observations suggest that large black holes may reside in the centers of some stellar clusters. X-ray observations in the last few years have shown unresolved sources in galaxies offset from their nuclei and with fluxes that, if isotropic, correspond to luminosities

of $L \approx 10^{39}$ to $10^{41} \mathrm{erg} \mathrm{s}^{-1}$ (e.g., Fabbiano, Schweizer, \& Mackie 1997; Colbert \& Mushotzky 1999; Matsumoto et al. 2001; Fabbiano, Zezas, \& Murray 2001). Many of these sources are associated with stellar clusters (Fabbiano et al. 1997; Angelini, Loewenstein, \& Mushotzky 2001). The strong variability observed in these sources suggests that they are black holes, and if the observed fluxes are neither strongly beamed nor super-Eddington, the implied 
masses are as high as $M \gtrsim 10^{3} M_{\odot}$. The fact the sources are non-nuclear implies masses $M \lesssim 10^{6} M_{\odot}$ since a larger mass would have rapidly sunk to the center of the host galaxy due to dynamical friction $\left(<10^{9} \mathrm{yr}\right.$ for a dispersion velocity of $100 \mathrm{~km} \mathrm{~s}^{-1}$ and a separation from the galaxy nucleus of $10^{2}$ pc as in the case of M82; Kaaret et al. 2001). In addition, optical observations of the globular clusters M15 and G1 show velocity profiles consistent with central black holes with masses of $2.5 \times 10^{3} M_{\odot}$ and $2.0 \times 10^{4} M_{\odot}$, respectively (Gebhardt et al. 2000; Gerssen et al. 2002; van der Marel, et al. 2002; Gebhardt, Rich, \& Ho 2002), although Baumgardt et al. (2003) demonstrate with their N-body simulations that the observations of G1 can be explained without a large black hole. Such intermediate-mass black holes (IMBHs) would be in a different mass category, and thus likely indicative of a different formation scenario, from either 3 - $20 M_{\odot}$ stellar-mass black holes, which are thought to be the result of core-collapse supernovae, or $10^{6}-10^{10} M_{\odot}$ supermassive black holes, which are found in the centers of many galaxies.

Several models have been proposed to account for the origin of IMBHs. Madau \& Rees (2001) and Schneider et al. (2002) suggest that they are the remnants of massive $\left(M \gtrsim 200 M_{\odot}\right)$ Population III stars. The low metallicity of these stars precludes cooling through metal line emission and enables them to reach masses much larger than ordinary main sequence stars. These large stars avoid significant mass loss due to stellar winds or pulsations, and the star may collapse to form a black hole with almost the same mass as the progenitor star. Portegies Zwart \& McMillan (2002) and Gürkan, Freitag, \& Rasio (2004) show with numerical simulations that the core of a young stellar cluster may collapse rapidly such that direct collisions of stars will lead to runaway growth of a single object with as much as $10^{-3}$ of the original cluster mass over the course of a few million years. Miller \& Hamilton (2002a) propose that over a Hubble time stellar-mass black holes in dense globular clusters may grow by mergers to the inferred IMBH masses. In their model, a black hole with mass greater than $50 M_{\odot}$ will interact with other massive objects to form binaries that will merge due to gravitational radiation. The merger process may proceed more quickly in the presence of encounters with a third black hole or another black hole binary (Miller \& Hamilton 2002b) if the encounters shrink the binary's orbit, as is known to happen with hard (tight) binaries (Heggie 1975).

Wherever and however IMBHs formed, the best candidates are found in stellar clusters where three-body encounters are important. An IMBH in a cluster, whether formed there or later swallowed by the cluster, will find its way to the center. As all of the heaviest objects in a cluster sink to the center in a process known as mass segregation, the IMBH will interact primarily with other massive objects and binaries (Sigurdsson \& Hernquist 1993; Fregeau et al. 2002). A single IMBH will tend to acquire companions through exchanges with binaries because the most massive pair of objects in a three-body encounter preferentially end up in 
the binary (e.g., Heggie, Hut, \& McMillan 1996). The IMBH binary will encounter other objects in the dense center of its host cluster, harden further, and ultimately merge.

These events are important sources of gravitational waves. The Advanced LIGO (Laser Interferometer Gravitational Wave Observatory) detector is expected to be capable of detecting mergers of IMBHs with $M \lesssim 100 M_{\odot}$ (Barish 2000), and LISA (Laser Interferometer Space Antenna) is expected to detect the earlier inspiral phase of an IMBH merger (Danzmann 2000). In order to predict the gravitational wave signature of the inspiral, the expected separations and eccentricities of the binaries must be known. Because three-body encounters alter the orbital parameters, simulations are needed to predict their distributions as well as the source population and event rates.

The three-body problem has been studied extensively, but with every new generation of computing power, our understanding of the problem advances with a wider range of numerical simulations and a changing perspective on this rich but conceptually simple problem. Previous studies of the three-body problem have tended to focus on the case of equal or nearly equal masses (e.g., Heggie 1975; Hut \& Bahcall 1983) though other mass ratios have been studied (e.g., Fullerton \& Hills 1982; Sigurdsson \& Phinney 1993; Heggie et al. 1996). The nearly equal mass case does not apply to the case of an IMBH in the core of a stellar cluster. In addition the vast majority of previous work has studied the effect of a single encounter on a binary. To determine the ultimate fate of an IMBH, simulations of sequences of encounters are needed. Furthermore, to our knowledge no previous work has considered the effects of orbital decay due to gravitational radiation between encounters, which we expect to be important for very tight binaries.

In this paper we present numerical simulations of sequences of high-mass ratio binarysingle encounters. We describe the code used to simulate the encounters in $\S 2$. Next, we present results of the simulations of sequences of encounters on a range of mass ratios with Newtonian gravity (§3.1) and with gravitational radiation between encounters $(\S 3.2)$ and show that including gravitational radiation decreases the duration of the sequence by $\sim 30$ to $40 \%$. In $\S 4$ and $\S 5$ we discuss the implications of these results for IMBH formation and gravitational wave detection.

\section{Numerical Method}

We perform numerical simulations of the interactions of a massive binary in a stellar cluster. Simulating the full cluster is beyond current N-body techniques, so we focus instead on a sequence of three-body encounters. Massive cluster objects, such as IMBHs and tight 
binary systems, tend to sink the centers of clusters so that a single IMBH is very likely to meet a binary (Sigurdsson \& Phinney 1995). Exchanges in which the IMBH acquires a close companion are common. Such a binary in a stellar cluster core will experience repeated interactions with additional objects as long as the recoils from these interactions do not eject the binary. Therefore, we simulate a sequence of encounters between a hard binary and an interloper. We perform one interaction and then use the resulting binary for the next encounter. This is repeated multiple times until the binary finally merges due to gravitational radiation. Because typical velocities involved are non-relativistic and the black holes are tiny compared to their separations, they are treated as Newtonian point masses. In order to test the influence of the binary's mass, we use a range of binary mass ratios. To simplify the problem we study a binary with mass ratio of $N: 10 M_{\odot}$ and a $10 M_{\odot}$ interloper, designated as $N: 10: 10$, and vary $N$ between $10 M_{\odot}$ and $10^{3} M_{\odot}$.

The simulations were done using a binary-single scattering code that was written to be as general purpose as possible. Because of the vast parameter space that needs to be covered, the code uses a Monte Carlo initial condition generator. The orbits are integrated using hnbody, a hierarchical, direct N-body integrator, with the adaptive fourth order Runge Kutta integrator option (K. Rauch \& D. Hamilton, in preparation) ${ }^{1}$. Because we focus on close approaches where a wide range of timescales are important, an adaptive scheme is often better than symplectic methods.

In wide hierarchical triples, direct integration can consume a large amount of computational time. To reduce this, we employ a two-body approximation scheme that tracks the phase of the inner binary. For a sufficiently large outer orbit, the orbit is approximately that of an object about the center of mass of the binary. We calculate this approximate two-body orbit analytically and keep track of the inner binary's phase. When the outer object nears the binary again, we revert to direct numerical integration.

The orbit is integrated until one of three conditions is met: 1) one mass departs along a hyperbolic path, 2) the system forms a hierarchical triple with outer semimajor axis greater than $2000 \mathrm{AU}$, an orbit so large that it would likely be perturbed in the high density of a cluster core and not return, or 3) the integration is prohibitively long, in which case the encounter is discarded and restarted with new randomly generated initial conditions. Roughly $10^{-4}$ of all encounters had to be restarted with most occurring for higher mass ratios where resonant encounters (encounters that have more than one close approach and are not simple fly-bys) are more common. In half of our simulations, we evolve the binary's orbit due to gravitational wave emission after each encounter. Since a binary in a cluster

\footnotetext{
${ }^{1}$ See http://janus.astro.umd.edu/HNBody/.
} 
spends most of its time and emits most of its gravitational radiation while waiting for an encounter rather than during an interaction, we only include gravitational radiation between encounters. To isolate this effect, we run simulations both with and without gravitational radiation. We include gravitational radiation by utilizing orbit-averaged expressions for the change in semimajor axis $a$ and eccentricity $e$ with respect to time (Peters 1964):

$$
\frac{d a}{d t}=-\frac{64}{5} \frac{G^{3} m_{0} m_{1}\left(m_{0}+m_{1}\right)}{c^{5} a^{3}\left(1-e^{2}\right)^{7 / 2}}\left(1+\frac{73}{24} e^{2}+\frac{37}{96} e^{4}\right)
$$

and

$$
\frac{d e}{d t}=-\frac{304}{15} \frac{G^{3} m_{0} m_{1}\left(m_{0}+m_{1}\right)}{c^{5} a^{4}\left(1-e^{2}\right)^{5 / 2}}\left(e+\frac{121}{304} e^{3}\right),
$$

where $m_{0}$ and $m_{1}\left(m_{0} \geq m_{1}\right)$ are the gravitational masses of the binary pair. Here $G$ is the gravitational constant, and $c$ is the speed of light. The orbital elements are evolved until the next encounter takes place, at a time that we choose randomly from an exponential distribution with a mean encounter time, $\left\langle\tau_{\text {enc }}\right\rangle=1 /\left\langle n v_{\infty} \sigma\right\rangle$, where $n$ is the number density of objects in the cluster's core, $v_{\infty}$ is the relative velocity, and $\sigma$ is the cross-section of the binary. If we assume the mass of the binary $m_{0}+m_{1} \gg m_{2}$, then

$$
\sigma \approx \pi r_{p}^{2}+4 \pi r_{p} G\left(m_{0}+m_{1}\right) / v_{\infty}^{2}
$$

where $r_{p}$ is the maximum considered close approach of $m_{2}$ to the binary's center of mass. For a thermal distribution of stellar speeds, $v_{\infty}=\left(m_{\mathrm{avg}} / m_{2}\right)^{1 / 2} v_{\mathrm{ms}}$, where $m_{\mathrm{avg}}=0.4 M_{\odot}$ is the average mass of the main sequence star and $v_{\mathrm{ms}}$ is the main sequence velocity dispersion. In our simulations, the second term of Eq. 3, gravitational focusing, dominates over the first. Averaging over velocity (assumed to be Maxwellian) we find

$$
\left\langle\tau_{\text {enc }}\right\rangle=2 \times 10^{7}\left(\frac{v_{\mathrm{ms}}}{10 \mathrm{~km} \mathrm{~s}^{-1}}\right)\left(\frac{10^{6} \mathrm{pc}^{-3}}{n}\right)\left(\frac{1 \mathrm{AU}}{r_{p}}\right)\left(\frac{1 M_{\odot}}{m_{0}+m_{1}}\right)\left(\frac{1 M_{\odot}}{m_{2}}\right)^{1 / 2} \mathrm{yr} .
$$

We then subject the binary to another encounter using orbital parameters adjusted by both the previous encounter and the gravitational radiation emitted between the encounters. This sequence of encounters continues until the binary merges due to gravitational wave emission. If orbital decay is not being calculated, then we determine that the binary has merged when the randomly drawn encounter time is longer than the timescale to merger, which is approximately

$$
\tau_{\text {merge }} \approx 6 \times 10^{17} \frac{\left(1 M_{\odot}\right)^{3}}{m_{0} m_{1}\left(m_{0}+m_{1}\right)}\left(\frac{a}{1 \mathrm{AU}}\right)^{4}\left(1-e^{2}\right)^{7 / 2} \mathrm{yr}
$$


for the high eccentricities of importance in this paper.

Global energy and angular momentum are monitored to ensure accurate integration. The code also keeps track of the duration of encounters, the time between encounters, changes in semimajor axis and eccentricity, and exchanges (events in which the interloping mass replaces one of the original members of the binary and the replaced member escapes).

As a test of our code, we compared simulations of several individual three-body encounters to compare with the work of Heggie et al. (1996). As part of a series of works examining binary-single star scattering events, Heggie et al. (1996) performed numerical simulations of very hard binaries with a wide range of mass ratios and calculated their cross-sections for exchange. We ran simulations of one encounter each of a sample of mass ratios for comparison. To facilitate comparison of encounters with differing masses, semimajor axes, and relative velocities of hard binaries, Heggie et al. (1996) use a dimensionless cross-section,

$$
\bar{\sigma}=\frac{2 v_{\infty}^{2} \Sigma}{\pi G\left(m_{0}+m_{1}+m_{2}\right) a},
$$

where $v_{\infty}$ is the relative velocity of the interloper and the binary's center of mass at infinity and $\Sigma$ is the physical cross-section for exchanges. We calculate $\Sigma$ as the product of the fraction of encounters that result in an exchange $\left(f_{\text {ex }}\right)$ and the total cross-section of encounters considered: $f_{\mathrm{ex}} \pi b_{\max }^{2}$, where $b_{\max }$ is an impact parameter large enough to encompass all exchange reactions. Our cross-sections are in agreement with those of Heggie et al. (1996) within the combined statistical uncertainty as seen in Table 1.

\section{Simulations and Results}

We used our code to run numerical experiments of three-body encounter sequences with a variety of mass ratios. The binaries consisted of a dominant body with mass, $m_{0}=10,20$, $30,50,100,200,300,500$, or $1000 M_{\odot}$ and a secondary of mass $m_{1}=10 M_{\odot}$. Because of mass segregation, the objects that the binary encounters will be the heaviest objects in the cluster. In order to simplify the problem, we consider only interactions with interlopers of mass $m_{2}=10 M_{\odot}$. The binary starts with a circular $a=10 \mathrm{AU}$ orbit, and the interloper has a relative speed at infinity of $v_{\infty}=10 \mathrm{~km} \mathrm{~s}^{-1}$ and an impact parameter, $b$, relative to the center of mass of the binary such that the pericenter distance of the hyperbolic encounter would range from $r_{p}=0$ to $5 a$. For all binaries, $v_{\text {circ }}=\left[G\left(m_{0}+m_{1}\right) / a\right]^{1 / 2} \geq 40 \mathrm{~km} \mathrm{~s}^{-1} \gg$ $v_{\infty}$, and thus all are considered hard. The Monte Carlo initial condition generator distributes the orientations and directions of encounters isotropically in space, and the initial phase of the binary is randomized such that it is distributed equally in time. We assume the cluster 
core has a density of $n=10^{5} \mathrm{pc}^{-3}$ and an escape velocity of $v_{\text {esc }}=50 \mathrm{~km} \mathrm{~s}^{-1}$ for the duration of the simulation. We discuss the consequences of changing the escape velocity in $\S$ 4. For each mass ratio, we simulate 1000 sequences with and without gravitational radiation between encounters.

\subsection{Pure Newtonian Sequences}

Figure 1a shows the change of semimajor axis and pericenter distance as a function of time over the course of a typical Newtonian sequence. The encounters themselves take much less time then the period between encounters, so a binary spends virtually all its time waiting for an interloper. Most of the time in this example is spent hardening the orbit from $1 \mathrm{AU}$ to 0.4 AU because as the binary shrinks, its cross-section decreases and the timescale to the next encounter increases. Figure $1 \mathrm{~b}$ shows the same sequence plotted as a function of number of encounters. The semimajor axis decreases by a roughly constant factor with each encounter. This is expected for a hard binary, which, according to Heggie's Law (Heggie 1975), tends to harden with each encounter at a rate independent of its hardness. The eccentricity and therefore the pericenter distance, $r_{p}=a(1-e)$, however, can change dramatically in a single encounter (for a discussion on eccentricity change of a binary in a cluster, see Heggie $\&$ Rasio 1996). This sequence ends with a very high eccentricity $(e=0.968)$, which reduces the merger time given by Eq. 5 to less than $\tau_{\text {enc }}$.

Table 2 summarizes our main results and shows a number of interesting trends. The average number of encounters per sequence, $\left\langle n_{\mathrm{enc}}\right\rangle$, increases with increasing mass ratio since the energy that the interloper can carry away scales as $\Delta E / E \sim m_{1} /\left(m_{0}+m_{1}\right)$ (Quinlan 1996) and since $n_{\mathrm{enc}} \sim E / \Delta E$ for a constant eccentricity. Energy conservation assures that every hardening event results in an increased relative velocity between the binary and the single black hole. If the velocity of the single black hole relative to the barycenter, and thus the globular cluster, is greater than the escape velocity of the cluster core (typically $v_{\text {esc }}=50 \mathrm{~km} \mathrm{~s}^{-1}$ for a dense cluster; see Webbink 1985), then the single mass will be ejected from the cluster. The average number of ejected masses per sequence, $\left\langle n_{\mathrm{ej}}\right\rangle$, also increases with increasing mass ratio because the higher mass ratio sequences have a larger number of encounters and because the larger mass at a given semimajor axis has more energy for the interloper to tap. Conservation of momentum guarantees that when a mass is ejected from the cluster at very high velocity, the binary may also be ejected. Table 2 lists $\left\langle f_{\text {binej }}\right\rangle$, the fraction of sequences that result in the ejection of the binary from the cluster. As expected, the fraction decreases sharply with increasing mass such that virtually none of the binaries with mass greater than $300 M_{\odot}$ escape the cluster. 

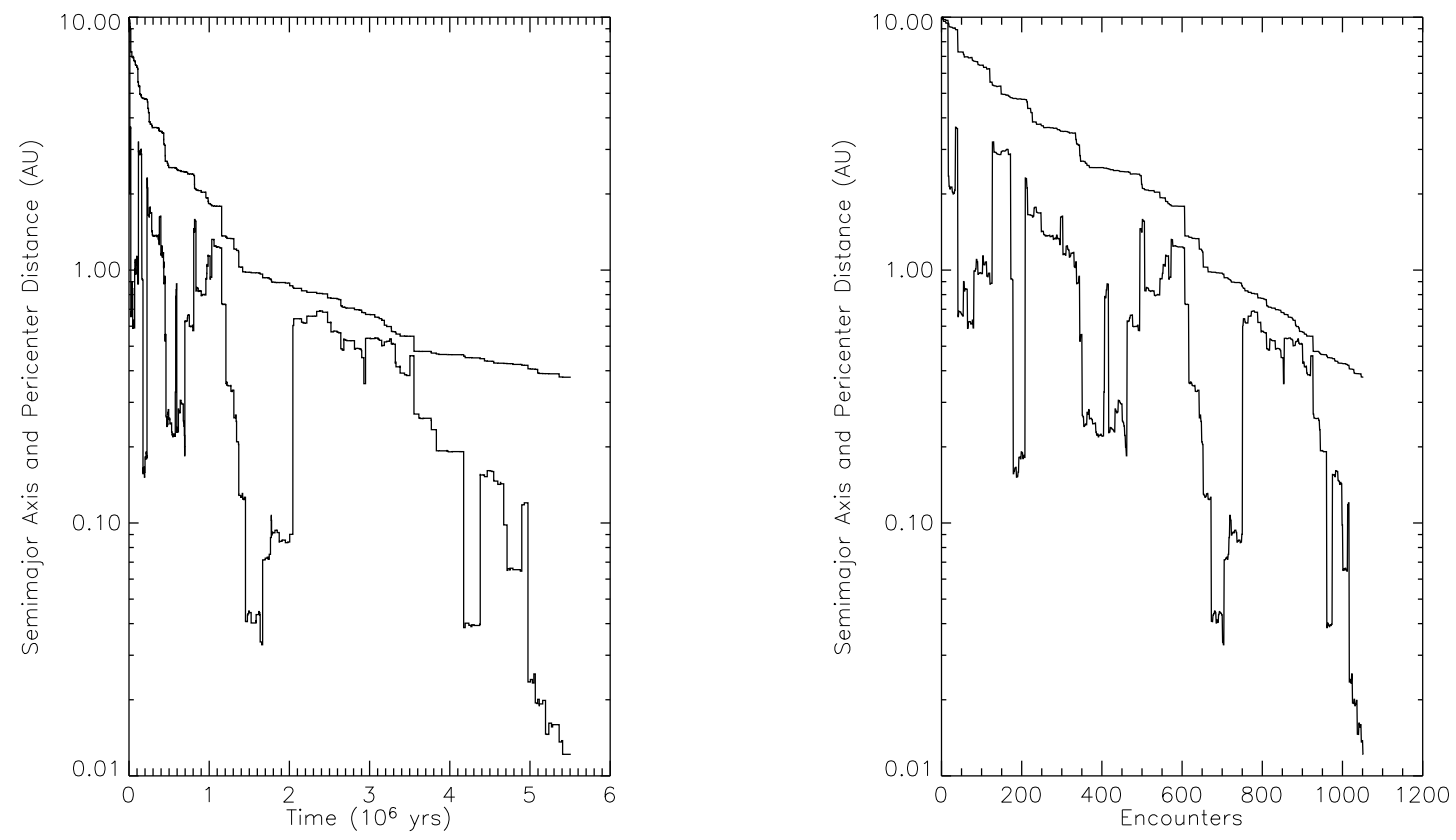

Fig. 1.- Newtonian 1000:10:10 sequence. These panels show the semimajor axes (upper lines) and pericenter distances (lower lines) as functions of time (left panel) and number of encounters (right panel) for one sequence of encounters with no gravitational wave emission. Each change in $a$ and $r_{p}$ is the result of a three-body encounter. Since the binary is hard, the semimajor axis gradually tightens by a roughly constant fractional amount per encounter with most of the time spent hardening the final fraction when close encounters are rare. The pericenter distance, however, fluctuates greatly due to large changes in eccentricity during a single encounter. The sequence ends at a very high eccentricity when the binary would merge due to gravitational radiation before the next encounter. 
The shape and size of the orbit after its last encounter determine the dominant gravitational wave emission during the inspiral and are of particular interest to us. The distribution of pre-merger semimajor axes for all mass ratios is shown in Figure 2. The distributions all have a similar shape that drops off at low a because the binary tends to merge before another encounter can harden it. For large orbits the binary will only merge for a high eccentricity, and thus there is a long tail in the histograms towards high $a$ from encounters that resulted in an extremely high eccentricity. The distributions for lower mass ratios are shifted to smaller $a$ because for a given orbit, a less massive binary will take longer to merge. This can also be seen in the mean final semimajor axis, $\left\langle a_{f}\right\rangle$, in Table 2.

Figure 3 shows the distribution of binary eccentricities after the final encounter for one mass ratio. The plot is strongly peaked near $e=1$, a property shared by all other mass ratios. This distribution is definitely not thermal, which would have a mean eccentricity $\langle e\rangle_{\text {th }} \approx 0.7$. The high eccentricity before merger results from both the strong dependence of merger time on eccentricity and the fact that the eccentricity can change drastically in a single encounter (see Fig. 1). As the semimajor axis decreases by roughly the same fractional amount in each encounter, the eccentricity increases and decreases by potentially large amounts with each strong encounter. When the eccentricity happens to reach a large value, the binary will merge before the next encounter. Figure 4 shows the eccentricity distribution for all encounters after the first 10 for all 1000 sequences with a mass ratio of 1000:10:10. The distribution is roughly thermal up to high eccentricity where the binaries merge. Thus merger selectively removes high eccentricity binaries from a thermal distribution.

\subsection{General Relativistic Binary Evolution}

The addition of gravitational radiation between Newtonian encounters is expected to alter a sequence since it is an extra source of hardening and since it circularizes the binary. Figure 5 shows a typical sequence for the 1000:10:10 mass ratio including gravitational radiation. Three-body interactions drive the binary's eccentricity up to $e=0.959$ and its semimajor axis down to $a=0.713 \mathrm{AU}$. Then starting at $t=2.2 \times 10^{6}$ yr over the course of about ten interactions that only weakly affect the eccentricity and semimajor axis, gravitational radiation causes the orbit to decay to $a=0.550 \mathrm{AU}$ and $e=0.946$ while the pericenter distance remains roughly constant. The corresponding semimajor axis change in the Newtonian only sequences in Figure 1 takes 45 encounters and more than twice as long although one must be careful when comparing two individual sequences. Gravitational waves

make the most difference when the pericenter distance is small, which is guaranteed at the end of a sequence, but can also happen in the middle as Figure 5 shows. 

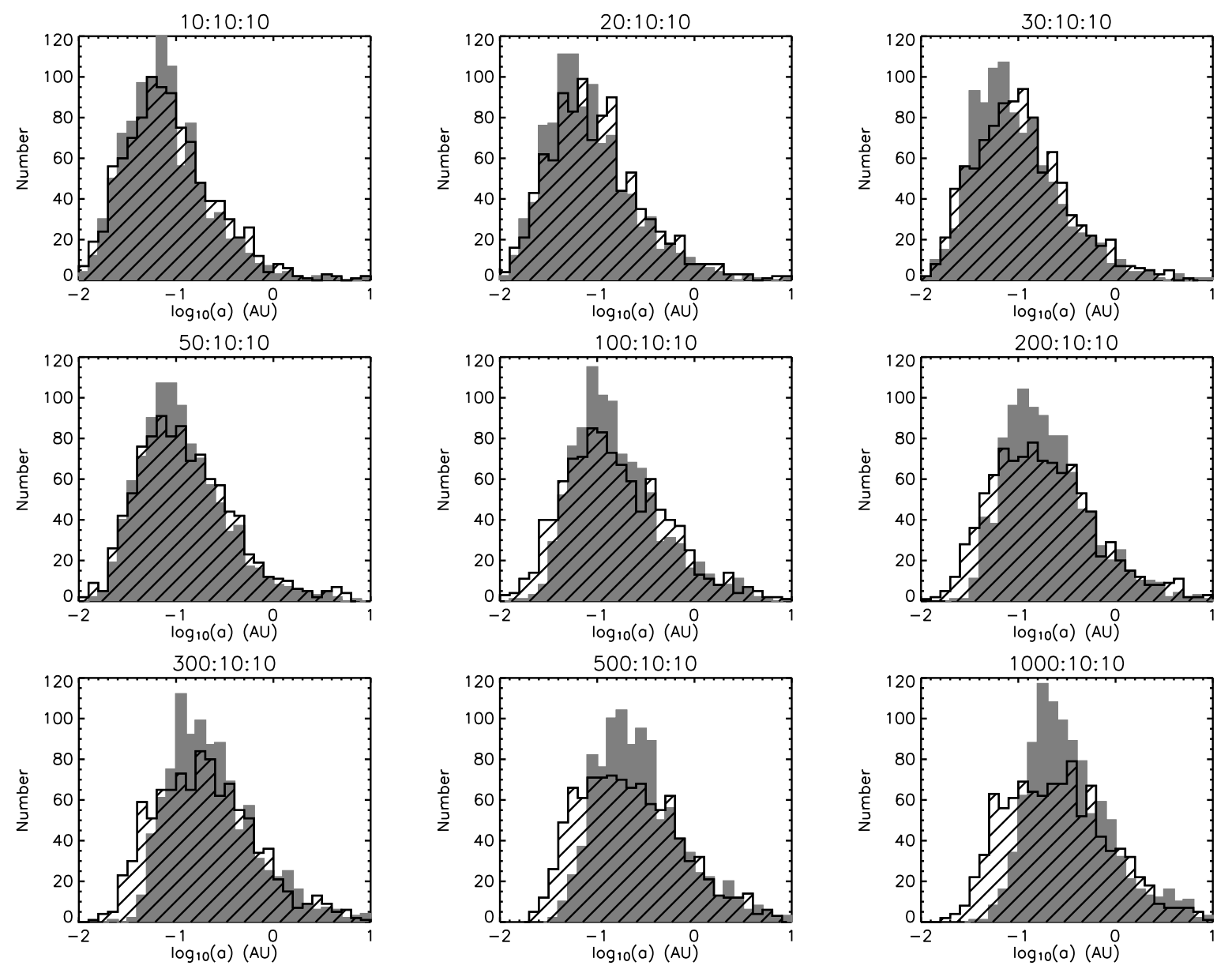

Fig. 2.- Histograms of final semimajor axes for all mass ratios. The solid histograms are pure Newtonian sequences, and the hatched histograms are sequences with gravitational radiation between encounters. The histograms all have similar shapes with a sharp drop at low a since the binary tends to merge before another encounter can harden it, and it has long tail at high $a$ where the binary will only merge with high eccentricity. The sequences with gravitational radiation have falloffs at smaller $a$ than those without due to both the circularization and the extra source of hardening. 


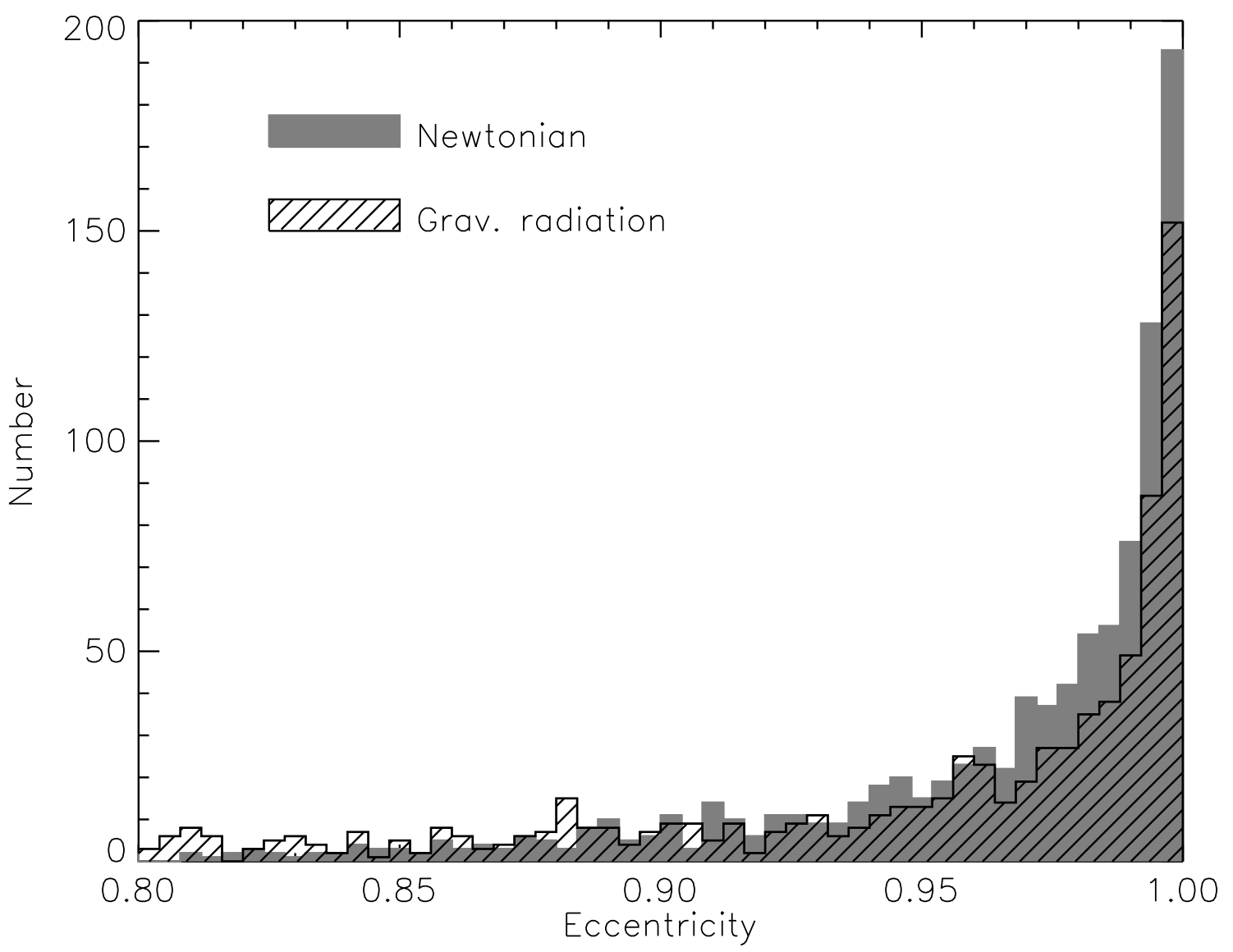

Fig. 3.- Histogram of final eccentricities for 1000:10:10 mass ratio. The solid histogram is from pure Newtonian sequences, and the hatched histogram is from sequences with gravitational radiation between encounters. The histogram is cut at $e=0.8$ because $e_{f}<0.8$ is rare. The histograms have roughly the same shape for both cases and for all mass ratios although the gravitational wave sequences have a consistently lower mean at higher mass ratios because gravitational wave emission damps eccentricities. The histograms show a decidedly non-thermal distribution and are strongly peaked near $e=1$. Because the timescale to merge due to gravitational radiation is so strongly dependent on $e$, the binary will merge when it happens to reach a high eccentricity. 


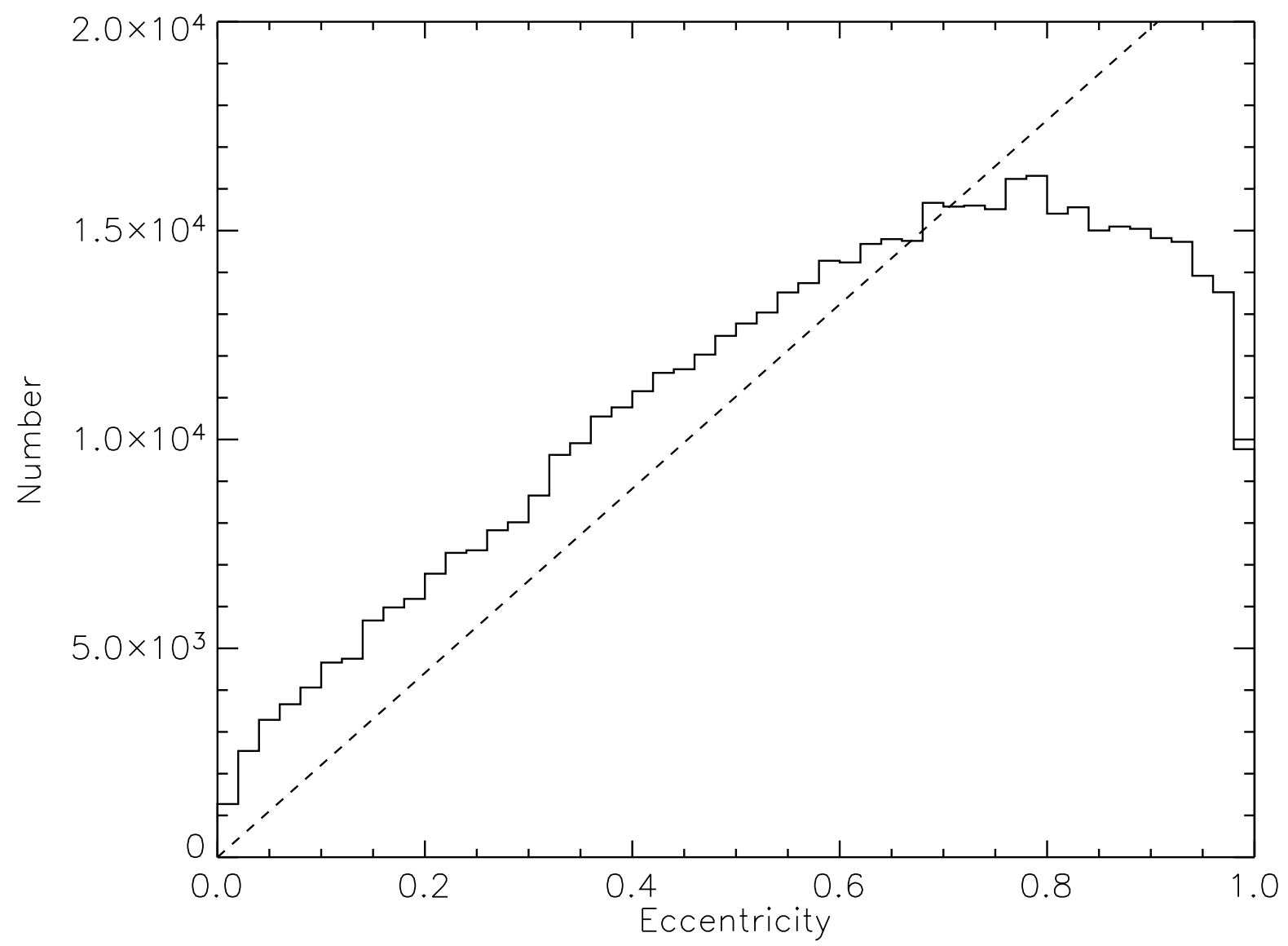

Fig. 4. - Solid line is a histogram of all eccentricities after each encounter except for the first ten for all pure Newtonian sequences of 1000:10:10. The dashed line is a thermal distribution of eccentricities. The distribution is roughly thermal for low eccentricity but deviates for $e \gtrsim 0.6$. The expected thermal distribution of eccentricities is altered by losses of high eccentricity orbits to merger. 


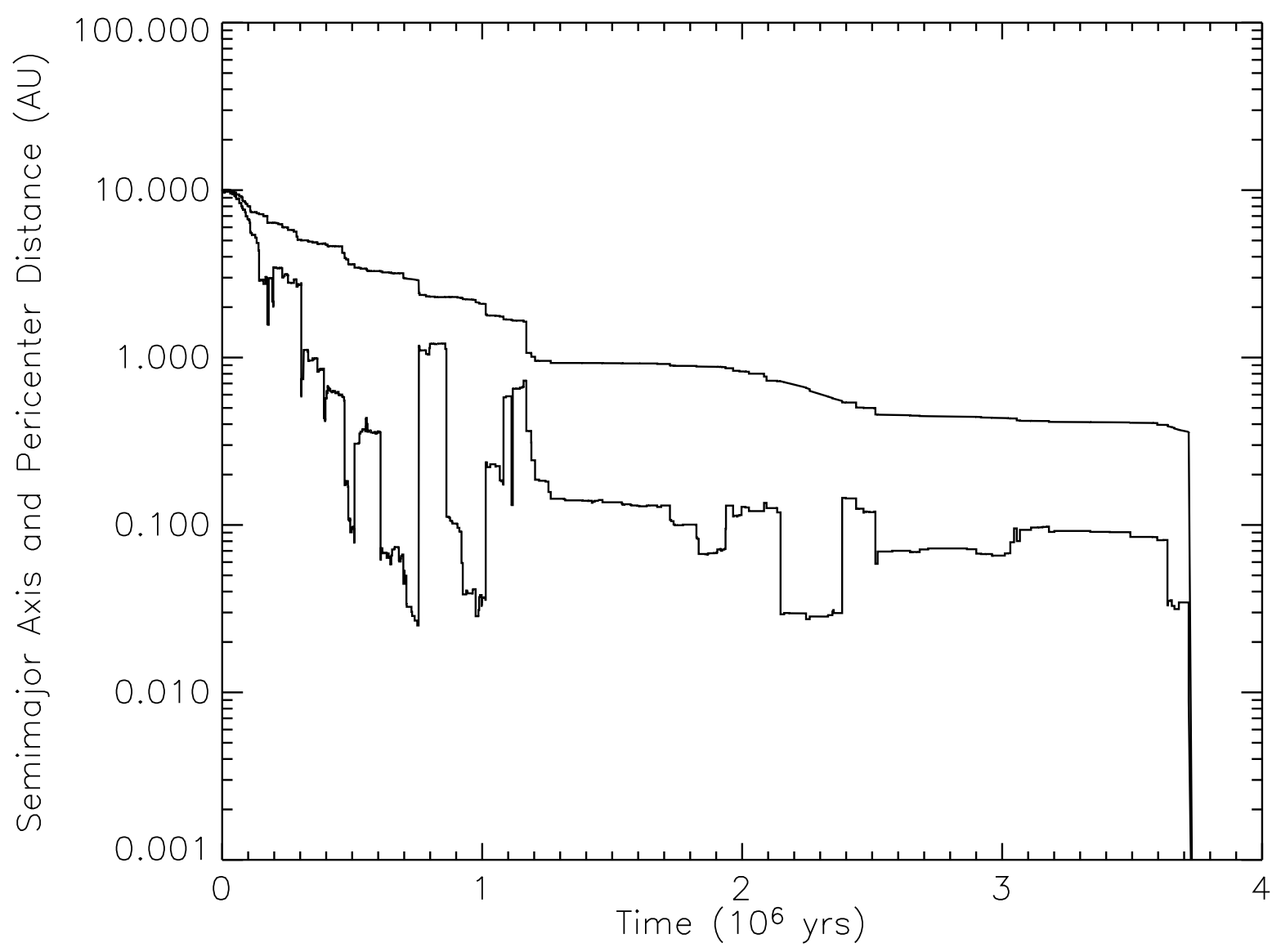

Fig. 5.- 1000:10:10 gravitational radiation sequence. Same as Figure 1a but for a sequence with gravitational radiation between encounters. The effects of gravitational radiation can be seen between 2.2 and $2.4 \times 10^{6}$ years. Over this period, the binary undergoes about ten interactions that do not significantly affect its orbit. During this time, the semimajor axis decays from $a=0.713 \mathrm{AU}$ to $0.550 \mathrm{AU}$ while the pericenter distance remains small and roughly constant. When an encounter reduces the eccentricity at $2.4 \times 10^{6}$ years, gravitational radiation is strongly reduced. Gravitational radiation becomes important again at the end of the sequence. The sequence ends with the binary's merger from gravitational waves. 
Table 2 summarizes the effect of adding gravitational radiation. In general the effect is greater at higher masses because gravitational radiation is stronger for a given orbit. Because of the extra energy sink, the binaries merge with fewer encounters, fewer black holes are ejected, and the fraction of sequences in which a binary is ejected is smaller. The most dramatic change is in the duration of the sequence, which gravitational radiation reduces by $27 \%$ to $40 \%$. The distributions of final semimajor axes (Fig. 2) and final eccentricities (Fig. 3) have similar shapes to the Newtonian only distributions. Due to the circularizing effect of gravitational radiation, binaries of all mass ratios merge with a smaller $\left\langle e_{f}\right\rangle$ than Newtonian only sequences with the largest difference at high mass ratios. Gravitational radiation also produces a smaller $\left\langle a_{f}\right\rangle$ for $m_{0} \gtrsim 300 M_{\odot}$. This can be seen in Figure 2 where the gravitational radiation simulations display an excess number of sequences with low $a_{f}$, which is a consequence of the binaries' lower $e_{f}$.

\section{Implications for IMBH Formation}

We can use these simulations to test the Miller \& Hamilton (2002a) model of IMBH formation. We assume that a $50 M_{\odot}$ seed black hole with a $10 M_{\odot}$ companion will undergo repeated three-body encounters with $10 M_{\odot}$ interloping black holes in a globular cluster with $v_{\text {esc }}=50 \mathrm{~km} \mathrm{~s}^{-1}$ and $n=10^{5} \mathrm{pc}^{-3}$. We also assume that the density of the cluster core remains constant as the IMBH grows. We then test whether the model of Miller \& Hamilton (2002a) can build up to IMBH masses, which we take to be $10^{3} M_{\odot}$, 1) without ejecting too many black holes from the cluster, 2) without ejecting the IMBH from the cluster, and 3) within the lifetime of the globular cluster. We also test how these depend on escape velocity and seed mass.

If the number of black holes ejected is greater than the total number of black holes in the cluster core, then the IMBH cannot build up to the required mass by accreting black holes alone. To calculate the total number of black holes ejected while building up to large masses, we sum the average number of ejections using a linear interpolation of the values in Table 2. Assuming a cluster escape velocity of $v_{\text {esc }}=50 \mathrm{~km} \mathrm{~s}^{-1}$, we find that the total number of black holes ejected when building up to $1000 M_{\odot}$ is approximately 6800 for our Newtonian only and 5300 for gravitational radiation simulations. This is far greater than the estimated $10^{2}$ to $10^{3}$ black holes available (Portegies Zwart \& McMillan 2000). If there were initially one thousand $10 M_{\odot}$ black holes in the cluster, mergers of the massive black hole with a series of $10 M_{\odot}$ black holes would exhaust half of the black holes in $\sim 2.6 \times 10^{8} \mathrm{yr}$ and would ultimately produce a $240 M_{\odot}$ black hole. Increasing the seed mass increases the final mass of the IMBH when half of the field black holes run out. If the seed mass were 
100, 200, or $300 M_{\odot}$, then the model would produce a 270,330 , or $410 M_{\odot}$ black hole after exhausting half of the cluster black hole population in $1.9,1.1$, or $0.8 \times 10^{8} \mathrm{yr}$, respectively. Figure 6 shows the number of black holes ejected as a function of initial black hole mass for a range of escape velocities. Growth times are much shorter than the $\sim 10^{9}$ yr necessary for stellar-mass black holes to eject each other from the cluster (Sigurdsson \& Hernquist 1993; Portegies Zwart \& McMillan 2000; J. M. Fregeau, S. A. Rappaport, \& V. Corless, in preparation; R. O'leary et al., in preparation). Therefore, self-depletion of stellar-mass black holes is not a limiting factor.

Of particular concern is whether the three-body scattering events will eject the binary from the cluster. The black hole can only merge with other black holes while it is in a dense stellar environment. The probability of remaining in the cluster after one sequence is $P=1-\left\langle f_{\text {binej }}\right\rangle$. As can be seen in Table 2 , once the black hole has built up to $\sim 300 M_{\odot}$, it is virtually guaranteed to remain in the cluster. When starting with $50 M_{\odot}$, we calculate the total probability of building up to $300 M_{\odot}$ to be 0.0356 . Figure 7 shows the probability of building up to $300 M_{\odot}$ as a function of starting mass for different escape velocities for the gravitational radiation case. Table 3 lists probabilities for selected seed masses and escape velocities for the gravitational radiation case.

In a similar manner, we calculate the total time to build up to $1000 M_{\odot}$, assuming that the supply of stellar-mass black holes and density remain constant, an assumption which leads to an underestimation of the time. While the time per merger is larger for the smaller masses, the total time is dominated at the higher masses since more mergers are needed for the same fractional increase in mass. For Newtonian only simulations the total time is $1.1 \times 10^{9} \mathrm{yr}$, and for simulations with gravitational radiation the total time is $7.1 \times 10^{8} \mathrm{yr}$. These are much less than the age of the host globular clusters. Figure 8 shows the time to reach a specified mass for both the Newtonian and gravitational radiation cases.

Although there is clearly enough time to build IMBHs as Miller \& Hamilton (2002a) propose, the issues of whether there are enough stellar-mass black holes and whether the cluster will hold onto the IMBH remain. The combination of an initial mass of $50 M_{\odot}$ and an escape velocity of $50 \mathrm{~km} \mathrm{~s}^{-1}$ is not likely to produce an IMBH in a globular cluster through three-body interactions with $10 M_{\odot}$ black holes, but the general process could still produce IMBHs. Miller \& Hamilton (2002a) argued that a seed mass of $50 M_{\odot}$ would be retained, but for analytical simplicity they assumed that every encounter changed the semimajor axis by the same fractional amount $\langle\Delta a / a\rangle$. Some encounters, however, can decrease the semimajor axis by several times the average value and thus impart much larger kicks. The authors therefore underestimated the minimum initial mass necessary to remain in the cluster. A hierarchical merging of stellar-mass black holes could, however, still produce an IMBH if 1) 


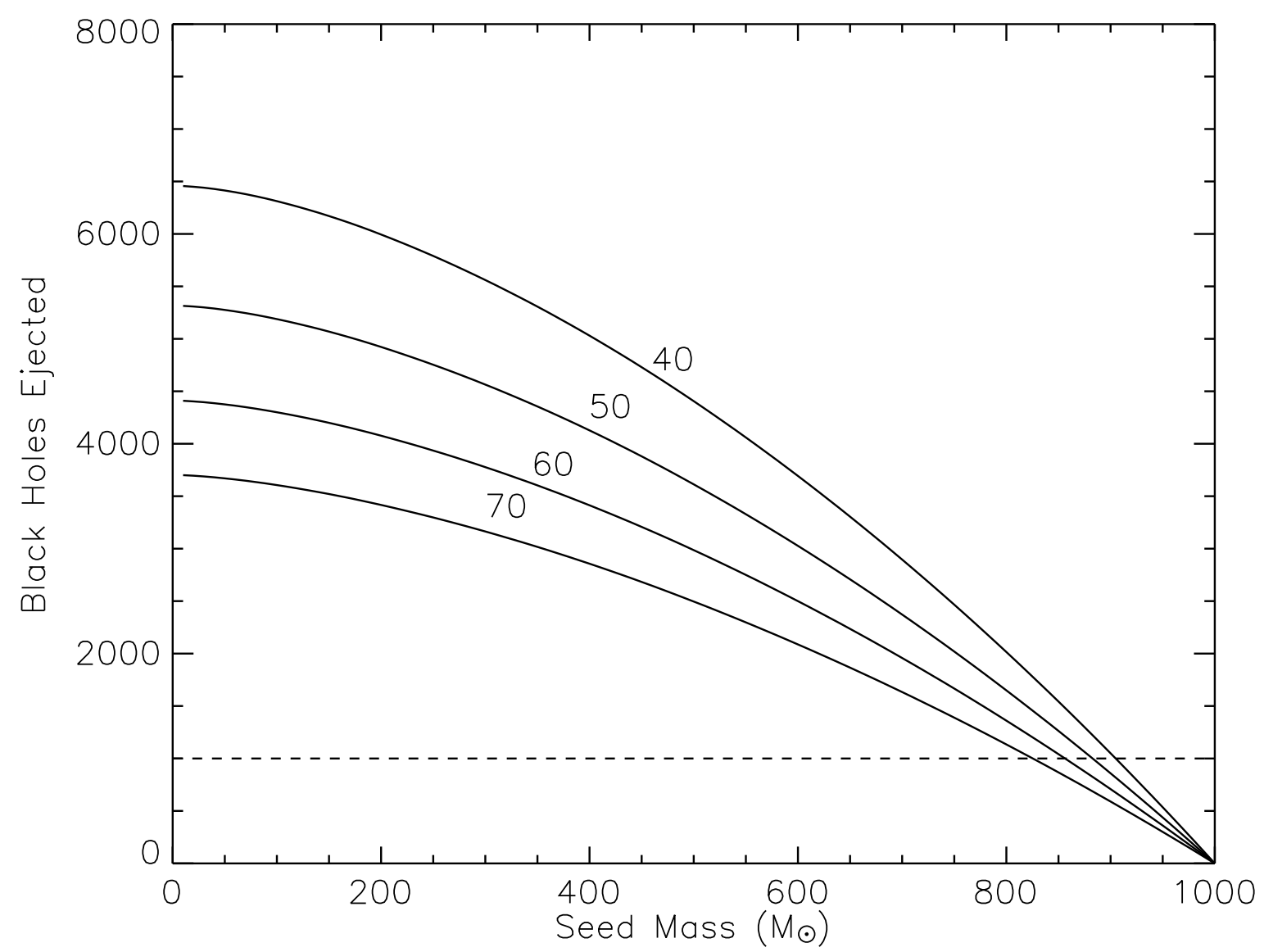

Fig. 6. - Plot of total number of black holes ejected in building up to $1000 M_{\odot}$ as a function of seed mass for the gravitational radiation case assuming different escape velocities. The four curves show different assumed cluster escape velocities in $\mathrm{km} \mathrm{s}^{-1}$. For all but the largest seed masses, the number of black holes ejected is greater than the estimated $\sim 10^{3}$ (indicated by the dashed line) present in a young globular cluster. 


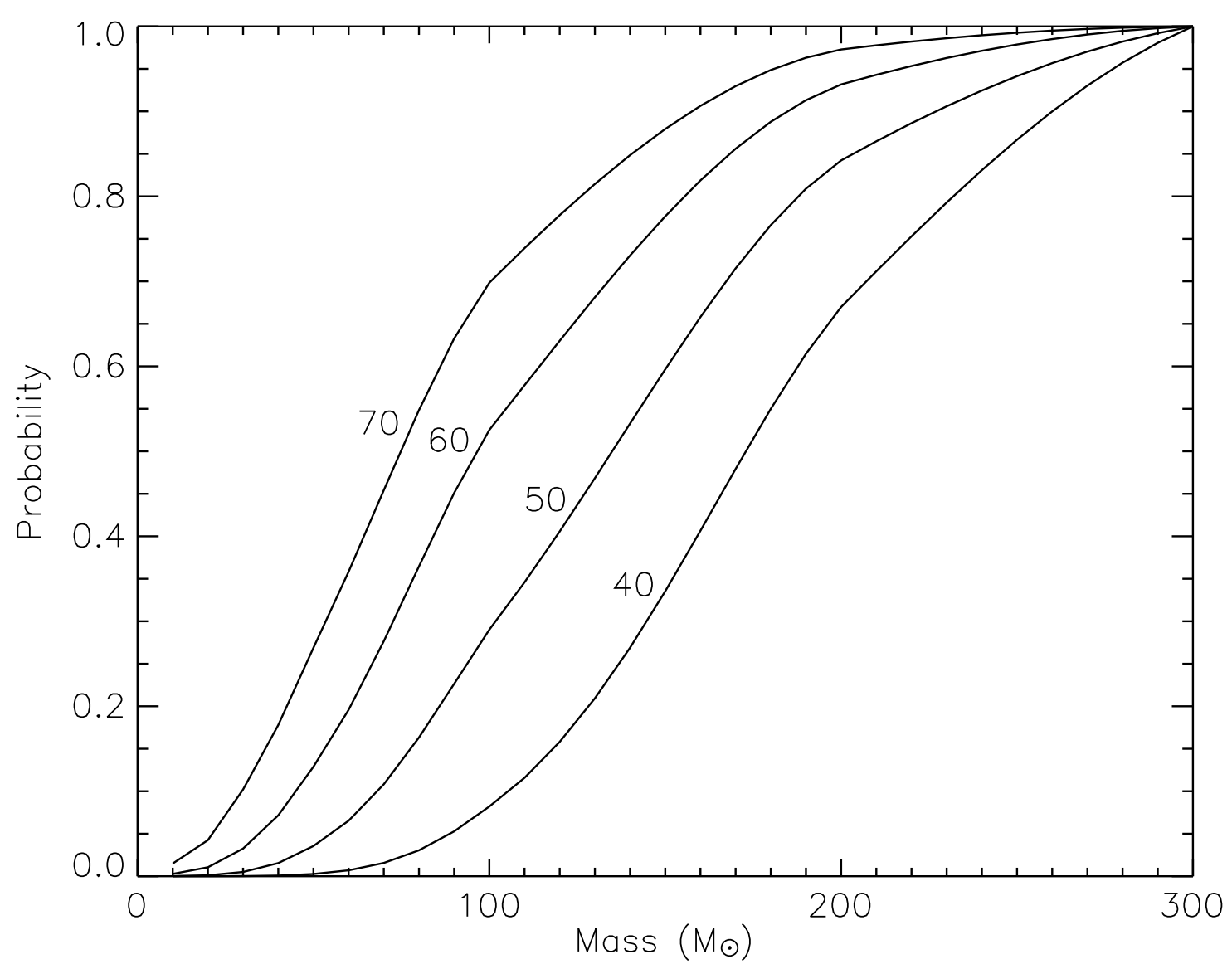

Fig. 7.- Plot of an IMBH's probability of remaining in the cluster and building up to $300 M_{\odot}$ as a function of starting mass of the dominant black hole for the gravitational radiation case assuming different escape velocities labeled in $\mathrm{km} \mathrm{s}^{-1}$. Once the black hole has built up to $300 M_{\odot}$ it is very unlikely that it will be ejected from the cluster. The lowest mass binaries are much more readily ejected and thus are very unlikely to survive a sequence of encounters. Miller \& Hamilton (2002a) suggest that IMBHs can be built in this manner with a starting mass $\approx 50 M_{\odot}$. We find that such small initial masses are likely to be ejected from the cluster core for reasonable escape velocities of dense clusters. 


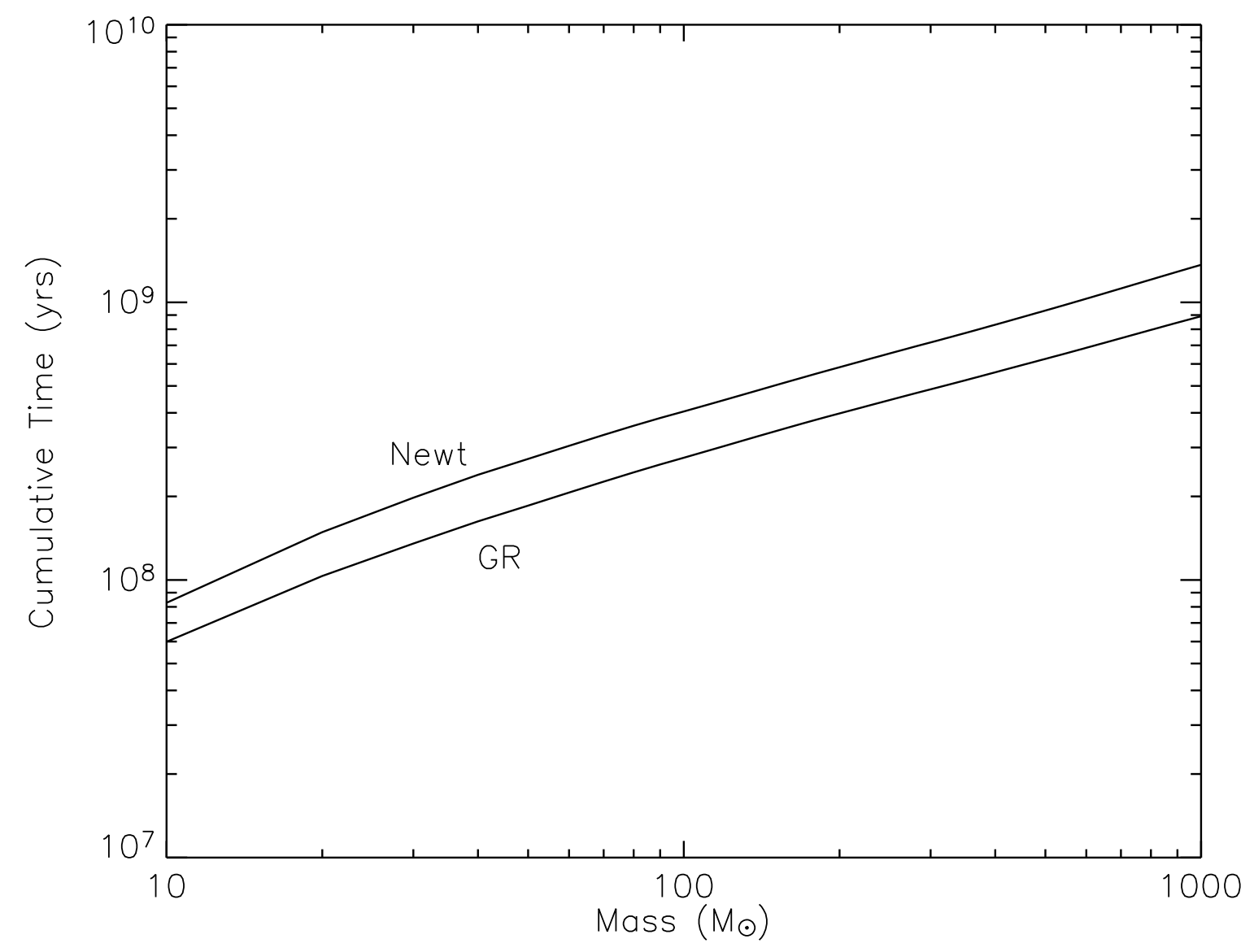

Fig. 8.- Plot of total time to build up to a certain mass when built by mergers with $10 M_{\odot}$ black holes for Newtonian only results and for runs with gravitational radiation between encounters. The Newtonian only simulations are slower to build up, but both cases reach $1000 M_{\odot}$ within about $10^{9}$ years. The time plotted assumes a constant density of black holes for the duration of IMBH formation. 
the initial mass of the black hole were were greater than $\left.50 M_{\odot}, 2\right)$ the escape velocity of the cluster were greater than $50 \mathrm{~km} \mathrm{~s}^{-1}$, or 3) additional dynamics were involved. We consider each of these in turn.

If the mass of the initial black hole were, e.g., $250 M_{\odot}$ before the onset of compact object dynamics, dynamical kicks would not be likely to eject the IMBH, and it would require fewer mergers to reach $1000 M_{\odot}$ and thus a smaller population of stellar mass black holes. The initial black hole could start with such a mass if it evolved from a massive Population III star or from a runaway collision of main sequence stars (Portegies Zwart \& McMillan 2002; Gürkan et al. 2004), or it could reach such a mass by accretion of young massive stars, which would be torn apart by tidal forces and impart little dynamical kick.

If the initial globular cluster mass is high enough (work by Meylan et al. 2001 indicate masses of $10^{7} M_{\odot}$ are available), then the cluster's gravity may be strong enough to retain the gas normally expelled by the first generation of supernovae. If that increases the escape velocity to, e.g., $v_{\text {esc }}=70 \mathrm{~km} \mathrm{~s}^{-1}$, the interactions result in a smaller fraction of ejected binaries. The probability of building from $50 M_{\odot}$ to $1000 M_{\odot}$ then increases by almost an order of magnitude.

In addition, processes with lower dynamical kicks could prevent ejection. One promising mechanism is the Kozai resonance (Kozai 1962; Miller \& Hamilton 2002b). If a stable hierarchical triple is formed, then resonant processes can pump up the inner binary's eccentricity high enough so that it would quickly merge due to gravitational radiation and without any dynamical kick to eject the IMBH from the cluster. Two-body captures (captures in which an interloper passes close enough to the isolated IMBH that it becomes bound and merges due to gravitational radiation) would also result in mergers without dynamical kicks. Both Kozai-resonance-induced mergers and two-body captures are devoid of dynamical kicks, but they would suffer a gravitational radiation recoil. A system in which a $10 M_{\odot}$ black hole merges into a $130 M_{\odot}$ non-rotating black hole would have a recoil velocity $20 \mathrm{~km} \mathrm{~s}^{-1} \leq v_{r} \leq 200 \mathrm{~km} \mathrm{~s}^{-1}$ (Favata, Hughes, \& Holz 2004). Since $v_{r} \sim\left(m_{1} / m_{0}\right)^{2}$, a merger between a $10 M_{\odot}$ black hole and a seed black hole of mass of $250 M_{\odot}$, as discussed above, would experience a recoil velocity $\lesssim 50 \mathrm{~km} \mathrm{~s}^{-1}$. Mergers with lower mass objects that are torn apart by tidal forces, such as white dwarfs, would receive no gravitational radiation recoil. Finally, a range of interloper masses instead of the simplified single mass population that we used here may also affect retention statistics since a smaller interloper would impart smaller kicks while still contributing to hardening.

Increasing the seed mass and the escape velocity will reduce the number of field black holes ejected but not by enough. As seen in Figure 6, using a seed mass $m_{0}=250 M_{\odot}$ and an escape velocity $v_{\text {esc }}=70 \mathrm{~km} \mathrm{~s}^{-1}$ reduces the number of black holes ejected by $40 \%$, but 
this is still several factors more than are available. The Kozai-resonance-induced mergers and two-body captures, however, are methods of merging without possibility of ejecting stellar-mass black holes. In order to reach our canonical $1000 M_{\odot}$ intermediate mass while ejecting fewer than $10^{3}$ black holes, $70-80 \%$ of the mergers must come from these ejectionless methods.

\section{Implications for Gravitational Wave Detection}

Our simulations make predictions interesting for gravitational wave detection. After the last encounter of a sequence, the binary will merge due to gravitational radiation. As the binary shrinks and circularizes, the frequency of the gravitational radiation emitted passes through the LISA band $\left(10^{-4}\right.$ to $\left.10^{0} \mathrm{~Hz}\right)$ (Danzmann 2000) and then through the bands of ground-based detectors such as LIGO, VIRGO, GEO-600, and TAMA (10 $10^{1}$ to $10^{3} \mathrm{~Hz}$ ) (Fidecaro et al. 1997; Schilling 1998; Barish 2000; Ando et al. 2002). By the time the binaries are detectable by ground-based instruments, they will have completely circularized, but while in the LISA band, some will have measurable eccentricities. We calculate the distribution of eccentricities detectable by LISA by integrating Equations 1 and 2 until the orbital frequency reaches $\nu_{\mathrm{orb}}=10^{-3} \mathrm{~Hz}$ at which point the gravitational wave frequency is in LISA's most sensitive range and is above the expected white dwarf background. Figure 9 shows the distribution of eccentricities for binaries with gravitational radiation in the LISA band. There are more low eccentricities at higher mass ratios. This is because at low mass ratios each encounter takes a fractionally larger amount of energy away from the binary than at high mass ratios. Thus at low mass ratios, the last encounter will tend to harden the binary such that it is closer to merger. At high mass ratios, however, encounters take a smaller fractional amount of energy from the binary, and, thus, the high mass ratio binaries have more time to circularize more during their orbital decay. For the 1000:10:10 mass ratio, a large fraction of the eccentricities are in the range $0.1 \lesssim e \lesssim 0.2$ where the binary is eccentric enough to display general relativistic effects such as pericenter precession, but circular templates may be sufficient for initial detection of the gravitational wave. Finally, because the first few hundred million years of a cluster's life witness a large number of mergers, recently formed and nearby super star clusters are promising sources of gravitational waves from IMBH coalescence. 

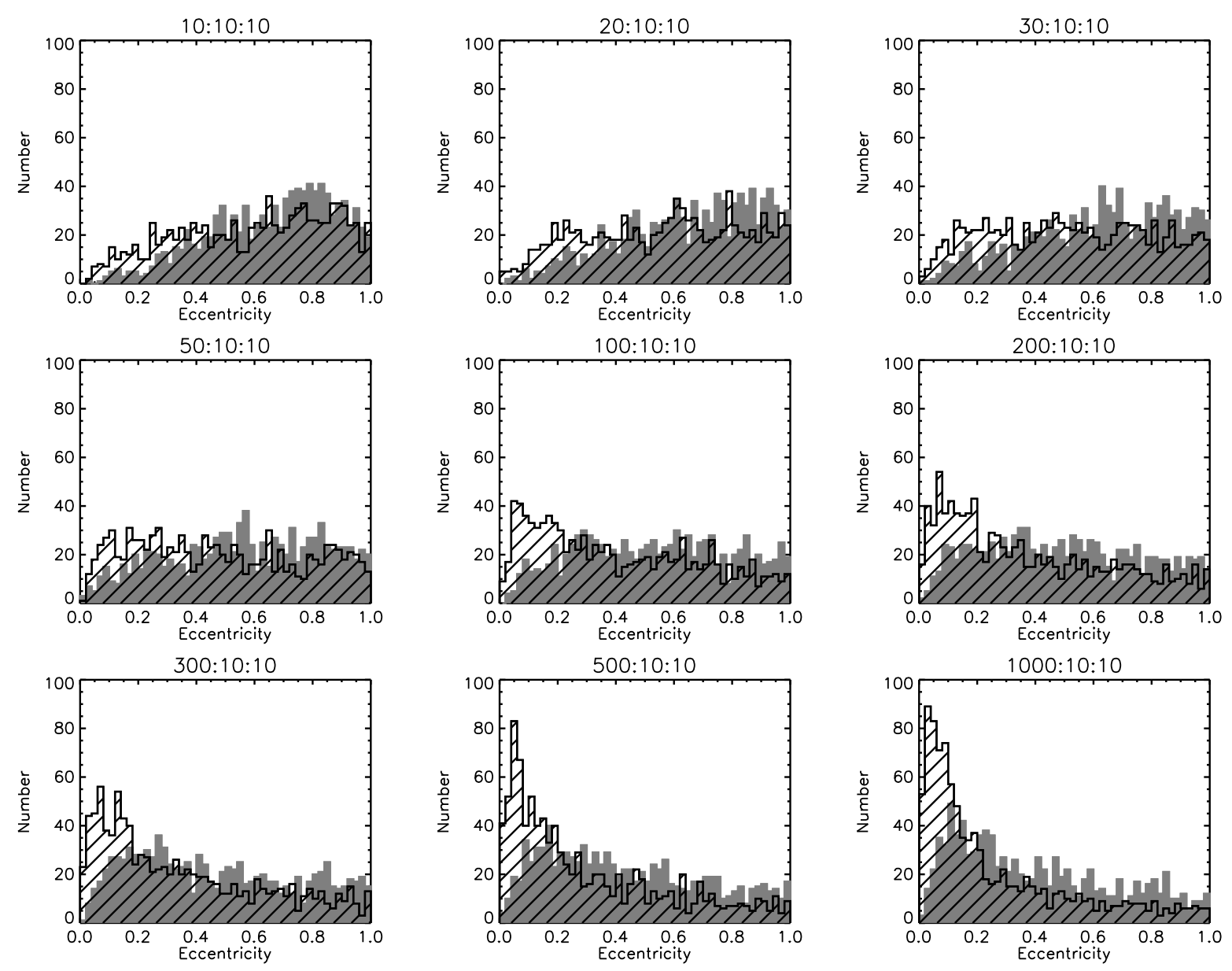

Fig. 9.- Distribution of eccentricities after integrating the Peters (1964) equations until in LISA band when the orbital frequency $\nu_{\text {orb }}=10^{-3} \mathrm{~Hz}$. The solid histograms are the Newtonian only sequences, and the hatched histograms are sequences with gravitational radiation. The sequences with gravitational radiation tend towards lower eccentricity since they have already started to circularize during the sequence. There is more difference between the two cases at higher mass ratios since gravitational radiation is stronger. Higher mass ratio binaries have lower eccentricities than lower mass ratio binaries since the latter start closer to merger after the final encounter. The 1000:10:10 mass ratio shows that a large number of detectable binaries would have $0.1 \lesssim e \lesssim 0.2$ such that they would likely be detectable by LISA with circular templates yet display measurable pericenter precession. 


\section{Conclusions}

We present results of numerical simulations of sequences of binary-single black hole scattering events in a dense stellar environment. We simulate three-body encounters until the binary will merge due to gravitational radiation before the next encounter. In half of our simulations, we include the effect of gravitational radiation between encounters.

1. Sequences of high mass ratio encounters. Our simulations cover a range of mass ratios including those corresponding to IMBHs in stellar clusters. Because the binaries simulated are tightly bound, the encounters steadily shrink the binary's semimajor axis until it merges. The eccentricity, however, jumps chaotically between high and low values over the course of a sequence. Merger usually occurs at high eccentricity since gravitational radiation is much stronger then.

2. Gravitational wave emission between encounters. The inclusion of gravitational radiation between encounters affects the simulations in several ways. The extra source of

shrinking caused by gravitational wave emission has the effect of shortening the sequence in terms of both the number of encounters and the total time, and the circularization from gravitational waves has the effect of decreasing the final eccentricity of the binary before it merges.

3. IMBH formation. Our simulations directly test the IMBH formation model of Miller \& Hamilton (2002a). We find that there is sufficient time to build up to $1000 M_{\odot}$ when starting from $50 M_{\odot}$, but our simulations also show that if there are a thousand $10 M_{\odot}$ black holes in the globular cluster, the seed black hole would only be able to grow to $240 M_{\odot}$ before exhausting half of the black holes in the cluster. In addition, the probability of the binary's remaining in the cluster during a growth from 50 to $240 M_{\odot}$ is small. In order to avoid ejection from the cluster with a reasonable probability, either the black hole must have a larger mass at the onset of dynamical encounters, the cluster's escape velocity must be larger, or the black hole must grow by some additional mechanisms such as by Kozairesonance-induced mergers, two-body captures, or from smaller interlopers.

4. Gravitational wave detection. The mergers of binary black hole systems are strong sources of detectable gravitational waves. We find that the merging binary will typically start with very high eccentricity. By the time the binary is detectable by the Advanced LIGO detector, it will have completely circularized, but when detectable by LISA, it may have moderate eccentricity $(0.1 \lesssim e \lesssim 0.2)$ such that it will display general relativistic effects such as pericenter precession and still possibly be detectable with circular templates. We find a high rate of mergers in the first few hundred million years of a globular cluster. This suggests that recently formed, nearby super star clusters are promising sources for 
gravitational radiation from IMBH coalescence.

Further work in this study will be to include a distribution of interloper masses instead of a single population of $10 M_{\odot}$ black holes. A mass distribution of black holes is a more realistic model of a cluster core and could change the outcomes of the sequences. Exchanges will be more important since encounters with the more prevalent smaller black holes may do most of the hardening until a more massive black hole exchanges into the binary.

We thank J. M. Fregeau, F. A. Rasio, and S. Sigurdsson for helpful discussions and comments. We are also grateful for the hospitality of the Center for Gravitational Wave Physics in which many fruitful ideas were born. Many of the results in this paper were obtained using the Beowulf cluster of the University of Maryland department of astronomy. This work was supported in part by NASA grant NAG 5-13229.

\section{REFERENCES}

Ando, M. et al. 2002, Classical Quantum Gravity, 19, 1409

Angelini, L., Loewenstein, M., \& Mushotzky, R. F. 2001, ApJ, 557, L35

Barish, B. C. 2000, Adv. Space Res., 25, 1165

Baumgardt, H., Makino, J., Hut, P., McMillan, S., \& Portegies Zwart, S. 2003, ApJ, 589, L25

Colbert, E. J. M., \& Mushotzky, R. F. 1999, ApJ, 519, 89

Danzmann, K. 2000, Adv. Space Res., 25, 1129

Fabbiano, G., Schweizer, F., \& Mackie, G. 1997, ApJ, 478, 542

Fabbiano, G., Zezas, A. L., \& Murray, S. S. 2001, ApJ, 554, 1035

Favata, M., Hughes, S. A., \& Holz, D. E. 2004 ApJ, submitted (astro-ph/0402056)

Fidecaro, F., et al. 1997, in Proc. 12th Italian Conf on General Relativity and Gravitational Physics, ed. M. Bassan, V. Ferrari, M. Francaviglia, F. Fucito, \& I. Modena (River Edge: World Scientific), 163

Fregeau, J. M., Joshi, K. J., Portegies Zwart, S. F., Rasio, F. A. 2002, ApJ, 570, 171

Fullerton, L. W., \& Hills, J. G. 1982, AJ, 87, 175 
Gebhardt, K., et al. 2000, ApJ, 543, L5

Gebhardt, K., Rich, R. M., \& Ho, L. C. ApJ, 578, L41

Gerssen, J., van der Marel, R. P., Gebhardt, K., Guhathakurta, P., Peterson, R. C., \& Pryor, C. 2002, AJ, 124, 3270

Gürkan, M. A., Freitag, M., \& Rasio, F. A. 2004, ApJ, in press (astro-ph/0308449)

Heggie, D. C. 1975, MNRAS, 173, 729

Heggie, D. C., Hut, P., \& McMillan, S. L. W. 1996, ApJ, 467, 359

Heggie, D. C., \& Rasio, F. A. 1996, MNRAS, 282, 1064

Hut, P., \& Bahcall, J. N. 1983, ApJ, 268, 319

Kaaret, P., Prestwich, A. H., Zezas, A., Murray, S. S., Kim, D.-W., Kilgard, R. E., Schelgel, E. M., \& Ward, M. J. 2001, MNRAS, 321, L29

Kozai, Y. 1962, AJ, 67, 591

Madau, P., Rees, M. J. 2001, ApJ, 551, L27

Matsumoto, H., Tsuru, T. G., Koyama, K., Awaki, H., Canizares, C. R., Kawai, N., Matsushita, S., \& Kawabe, R. 2001, ApJ, 547, L25

Meylan, G., Sarajedini, A., Jablonka, P., Djorgovski, S. G., Bridges, T., \& Rich, R. M. 2001 AJ, 122, 830

Miller, M. C., \& Hamilton, D. P. 2002, MNRAS, 330, 232

Miller, M. C., \& Hamilton, D. P. 2002, ApJ, 576, 894

Peters, P. C. 1964, Phys. Rev. B, 136, 1224

Portegies Zwart, S. F., \& McMillan, S. L. W. 2000, ApJ, 528, L17

Portegies Zwart, S. F., \& McMillan, S. L. W. 2002, ApJ, 576, 899

Quinlan, G. 1996, New Astronomy, 1, 35

Schilling, R. 1998, in AIP Conf. Proc. 456, Laser Interferometer Space Antenna, Second International LISA Symp., ed. W. M. Folkner (New York:AIP), 217

Schneider, R., Ferrara, A., Natarajan, P., \& Omukai, K. 2002, ApJ, 571, 30 
Sigurdsson, S., \& Hernquist, L. 1993, Nature, 364, 423

Sigurdsson, S., \& Phinney, E. S. 1993, ApJ, 415, 631

Sigurdsson, S., \& Phinney, E. S. 1995, ApJS, 99, 609

van der Marel, R. P. 2003 Carnegie Observatories Astrophysics Series, Vol. 1: Coevolution of Black Holes and Galaxies, ed. L. C. Ho (Cambridge: Cambridge Univ. Press), in press

van der Marel, R. P., Gerssen, J., Guhathakurta, P., Peterson, R. C., \& Gebhardt, K. 2002, AJ, 124, 3255

Webbink, R. F. 1985, in Dynamics of Star Clusters, IAU Symposium 113, ed. J. Goodman \& P. Hut (Dordrecht:Reidel), 541 
Table 1. Single Encounter Cross-sections for Exchange

\begin{tabular}{cccl}
\hline \hline$m_{0}: m_{1}: m_{2}$ & Ejected Mass & HHM96 & This Work \\
\hline $10: 1: 1$ & 1 & $1.054 \pm .105$ & $1.086 \pm .023$ \\
& 10 & - & - \\
$10: 1: 10$ & 1 & $7.825 \pm .360$ & $7.741 \pm .255$ \\
$3: 1: 1$ & 10 & $0.520 \pm .087$ & $0.513 \pm .043$ \\
& 1 & $2.311 \pm .170$ & $2.465 \pm .073$ \\
& 3 & $0.059 \pm .025$ & $0.072 \pm .007$ \\
\hline
\end{tabular}

Note. - This table compares dimensionless cross-sections for exchange $\bar{\sigma}$ (see text for details) calculated by Heggie et al. 1996 and by us. The first column lists the masses, with binary components $m_{0}$ and $m_{1}$. Column two shows the mass of the ejected object. The ejection of the smaller mass is energetically favored so it always has a larger cross-section. There is general agreement between the two calculations to within the statistical uncertainty, which we calculate as $\bar{\sigma} / N_{\mathrm{ex}}^{1 / 2}$, where $N_{\mathrm{ex}}$ is the total number of exchanges. 
Table 2. Sequence Statistics

\begin{tabular}{|c|c|c|c|c|c|c|c|}
\hline$m_{0}$ & Case & $\left\langle n_{\mathrm{enc}}\right\rangle$ & $\left\langle n_{\mathrm{ej}}\right\rangle$ & $\left\langle f_{\text {binej }}\right\rangle$ & $\left\langle t_{\text {seq }}\right\rangle / 10^{6} \mathrm{yr}$ & $\left\langle a_{f}\right\rangle / \mathrm{AU}$ & $\left\langle e_{f}\right\rangle$ \\
\hline \multirow[t]{2}{*}{10} & Newt. & 51.6 & 3.9 & 0.880 & 82.72 & 0.164 & 0.929 \\
\hline & GR Evol. & 48.7 & 3.7 & 0.839 & 59.89 & 0.190 & 0.901 \\
\hline \multirow[t]{2}{*}{20} & Newt. & 51.3 & 6.5 & 0.835 & 65.94 & 0.178 & 0.924 \\
\hline & GR Evol. & 47.1 & 6.1 & 0.776 & 43.46 & 0.230 & 0.898 \\
\hline \multirow[t]{2}{*}{30} & Newt. & 58.9 & 9.3 & 0.753 & 49.11 & 0.198 & 0.926 \\
\hline & GR Evol. & 55.1 & 8.6 & 0.676 & 31.89 & 0.222 & 0.892 \\
\hline \multirow[t]{2}{*}{50} & Newt. & 73.2 & 14.6 & 0.581 & 33.75 & 0.230 & 0.919 \\
\hline & GR Evol. & 66.7 & 13.0 & 0.455 & 22.73 & 0.285 & 0.892 \\
\hline \multirow[t]{2}{*}{100} & Newt. & 102.0 & 24.0 & 0.229 & 21.35 & 0.327 & 0.936 \\
\hline & GR Evol. & 93.4 & 20.1 & 0.161 & 14.97 & 0.357 & 0.873 \\
\hline \multirow[t]{2}{*}{200} & Newt. & 158.4 & 38.2 & 0.043 & 15.13 & 0.387 & 0.938 \\
\hline & GR Evol. & 140.3 & 31.5 & 0.026 & 9.998 & 0.444 & 0.872 \\
\hline \multirow[t]{2}{*}{300} & Newt. & 208.5 & 49.1 & 0.013 & 11.89 & 0.468 & 0.943 \\
\hline & GR Evol. & 184.0 & 39.4 & 0.006 & 7.822 & 0.445 & 0.874 \\
\hline \multirow[t]{2}{*}{500} & Newt. & 308.7 & 71.1 & 0.001 & 9.920 & 0.528 & 0.944 \\
\hline & GR Evol. & 269.1 & 54.9 & 0 & 6.225 & 0.488 & 0.860 \\
\hline \multirow[t]{2}{*}{1000} & Newt. & 562.4 & 117.3 & 0 & 7.363 & 0.641 & 0.953 \\
\hline & GR Evol. & 483.0 & 88.9 & 0 & 4.427 & 0.556 & 0.851 \\
\hline
\end{tabular}

Note. - Table 2 summarizes the main results of our simulations of sequences of threebody encounters. For each dominant mass, $m_{0}$, we ran 1000 sequences of pure Newtonian encounters (Newt.) and 1000 sequences of the more realistic Newtonian encounters with gravitational radiation between encounters (GR Evol.). The columns list the average number of encounters per sequence $\left\langle n_{\text {enc }}\right\rangle$, the average number of black holes ejected from the cluster in each sequence $\left\langle n_{\mathrm{ej}}\right\rangle$, the fraction of sequences in which the binary is ejected from the cluster, $\left\langle f_{\text {binej }}\right\rangle$, the average total time for the sequence $\left\langle t_{\text {seq }}\right\rangle$, the average final semimajor axis $\left\langle a_{f}\right\rangle$, and the average final eccentricity $\left\langle e_{f}\right\rangle$. 
Table 3. IMBH Formation

\begin{tabular}{ccccc}
\hline \hline $\begin{array}{c}\text { Seed Mass } \\
\left(M_{\odot}\right)\end{array}$ & $\begin{array}{c}v_{\text {esc }} \\
\left(\mathrm{km} \mathrm{s}^{-1}\right)\end{array}$ & $\begin{array}{c}\text { Probability to remain } \\
\text { in cluster }\end{array}$ & $\begin{array}{c}\text { Number of } \\
\text { BH ejections }\end{array}$ & $\begin{array}{c}\text { Time } \\
\left(10^{8} \mathrm{yr}\right)\end{array}$ \\
\hline \multirow{2}{*}{50.0} & 40.0 & 0.00264 & 6414 & 7.06 \\
& 50.0 & 0.0356 & 5276 & \\
& 60.0 & 0.129 & 4038 & \\
& 70.0 & 0.269 & 3573 & \\
\hline 100.0 & 40.0 & 0.0821 & 6312 & 6.15 \\
& 50.0 & 0.290 & 5188 & \\
& 60.0 & 0.525 & 3963 & \\
\hline 200.0 & 70.0 & 0.698 & 3606 & \\
& 40.0 & 0.670 & 5995 & 4.93 \\
& 50.0 & 0.842 & 4922 & \\
& 60.0 & 0.932 & 4077 & \\
\hline 300.0 & 70.0 & 0.978 & 3417 & \\
& 40.0 & 1.000 & 5561 & 4.05 \\
& 50.0 & 1.000 & 4564 & \\
& 60.0 & 1.000 & 3777 & \\
& 70.0 & 1.000 & 3164 & \\
\hline
\end{tabular}

Note. - This table lists values for selected seed masses and cluster escape velocities for the gravitational radiation case. Column 3 lists the probability for the IMBH to remain in the cluster until it reaches a mass of $300 M_{\odot}$. The fourth column lists the total number of black holes ejected in building up to $1000 M_{\odot}$. Column 5 lists the total time to build up to $1000 M_{\odot}$. The total time is not affected by the escape velocity because the density of black holes in the cluster core is taken to be constant. 